\title{
An Exactly Solved Model of Three Dimensional Surface Growth in the Anisotropic KPZ Regime
}

\author{
M. Prähofer*, H. Spohn ${ }^{\dagger}$ \\ Theoretische Physik, Ludwig-Maximilians-Universität, Theresienstr. 37, \\ D-80333 München, Germany
}

(March 22, 2018)

\begin{abstract}
We generalize the surface growth model of Gates and Westcott to arbitrary inclination. The exact steady growth velocity is of saddle type with principal curvatures of opposite sign. According to Wolf this implies logarithmic height correlations, which we prove by mapping the steady state of the surface to world lines of free fermions with chiral boundary conditions.
\end{abstract}

Keywords: Surface growth, logarithmic correlations, fermion world lines

\section{INTRODUCTION}

In its simplest form, deterministic surface growth is governed by

$$
\frac{\partial}{\partial t} h(\mathbf{x}, t)=v(\nabla h(\mathbf{x}, t))
$$

Here $h(\mathbf{x}, t)$ is the surface profile at time $t$ relative to a reference plane, $\mathbf{x} \in \mathbb{R}^{2}$, and (11) just expresses that the local velocity $v$ along $h$ depends only on the local slope $\mathbf{u}, \mathbf{u}=\nabla h$. Usually microscopic growth mechanisms, like deposition and evaporation of atoms, are noisy. Kardar, Parisi, and Zhang [i] modelled this randomness by adding noise and relaxation to (11). Expanding in the slope relative to $\mathbf{u}_{o}$, they obtained the KPZ equation (in $2+1$ dimensions)

$$
\frac{\partial}{\partial t} h=v\left(\mathbf{u}_{o}\right)+\sum_{i=1}^{2} \partial_{i} v\left(\mathbf{u}_{o}\right) \frac{\partial h}{\partial x_{i}}+\frac{1}{2} \sum_{i, j=1}^{2} \partial_{i} \partial_{j} v\left(\mathbf{u}_{o}\right) \frac{\partial h}{\partial x_{i}} \frac{\partial h}{\partial x_{j}}+\nu \Delta h+\xi
$$

with $\xi$ space-time white noise. If $\partial_{i} \partial_{j} v=\delta_{i, j} \lambda, \lambda \neq 0$, then the nonlinearity is of the standard form $\frac{1}{2} \lambda(\nabla h)^{2}$. The large space-time scaling behavior is then governed by a non-Gaussian strong coupling fixed point. We refer to [2 4] as surveys.

Wolf [5 noticed that the Taylor coefficient $\partial^{2} v$ is really a symmetric $2 \times 2$ matrix which allows for the possibility $\operatorname{det} \partial^{2} v \leq 0$. Based on a one-loop RG calculation he concluded that in this case the nonlinearity in (2) is irrelevant and the statistics of the growing surface is identical to the one of an equilibrium interface. In particular, in the steady state the height-height correlations grow logarithmically with distance.

At the time the prediction of Wolf came as a great surprise. In our paper we support his claim by a model surface for which we determine the growth velocity and the steady state correlations explicitly. Our model was proposed and studied by Gates and Westcott [6] who, in particular, computed the steady state. Since detailed balance is not available, there is no general method for obtaining the steady state. It is defined only implicitly as the invariant distribution of some stochastic evolution. Thus an explicit example is a most welcome exception. Gates and Westcott considered only tilts of the form $\mathbf{u}=\left(u_{1}, 0\right)$, which does not suffice to check the Wolf scenario. To extend their surface model to arbitrary tilt and to obtain its steady state is straightforward. One merely has to impose chiral boundary conditions for the height lines. Our main advance is to handle such boundary conditions in the limit of large volume through a transfer matrix which includes the "center of mass" coordinate. We compute the average velocity $v(\mathbf{u})$ and show that $\operatorname{det} \partial^{2} v \leq 0$ for arbitrary $\mathbf{u} \neq(0,0)$. To complete the argument we also determine the height-height correlations and verify their logarithmic behaviour. Presumably with some extra effort along the lines of [7] one could also show that the statistics is Gaussian on a large scale.

*email:praehofer@stat.physik.uni-muenchen.de

†email:spohn@stat.physik.uni-muenchen.de 
Let us define the Gates-Westcott model of a crystalline surface (see Figure 1). At a given time it is specified by the height profile $h(m, \tau)$. Here $m \in \mathbb{Z}, \tau \in \mathbb{R}$, and $h \in \mathbb{Z}$. If also $\tau \in \mathbb{Z}$, then the bulk crystal would be built up from unit cubes. Mathematically it is somewhat simpler to allow for real $\tau$. We assume that $h(m, \tau)$ is non increasing in $m$. Therefore we can introduce the collection of height lines $\left\{\phi_{j}(\tau)\right\}$ such that

$$
h(m, \tau)=J-j \quad \text { for } \quad \phi_{j}(\tau) \leq m<\phi_{j+1}(\tau) .
$$

$\phi_{j}$ and $\phi_{j+1}$ are the boundaries of the terrace with height $J-j$. We want to allow for arbitrary step heights along the discrete 1-direction. Formally, this means that we admit also a terrace width 0 . The height lines satisfy then the constraint $\phi_{j}(\tau) \leq \phi_{j+1}(\tau) \in \mathbb{Z}$ for all $\tau \in \mathbb{R}$. On the other hand along the continuous 2-direction we only admit step height 1 . Thus a height line $\phi_{j}$ is specified by its location, say at $\tau=0, \phi_{j}(0)$, and the location of the rightward steps (=kinks), $\phi_{j}\left(\tau_{-}\right)+1=\phi_{j}\left(\tau_{+}\right)$, and the leftward steps (=antikinks),$\phi_{j}\left(\tau_{-}\right)-1=\phi_{j}\left(\tau_{+}\right)$as in Figure 1.

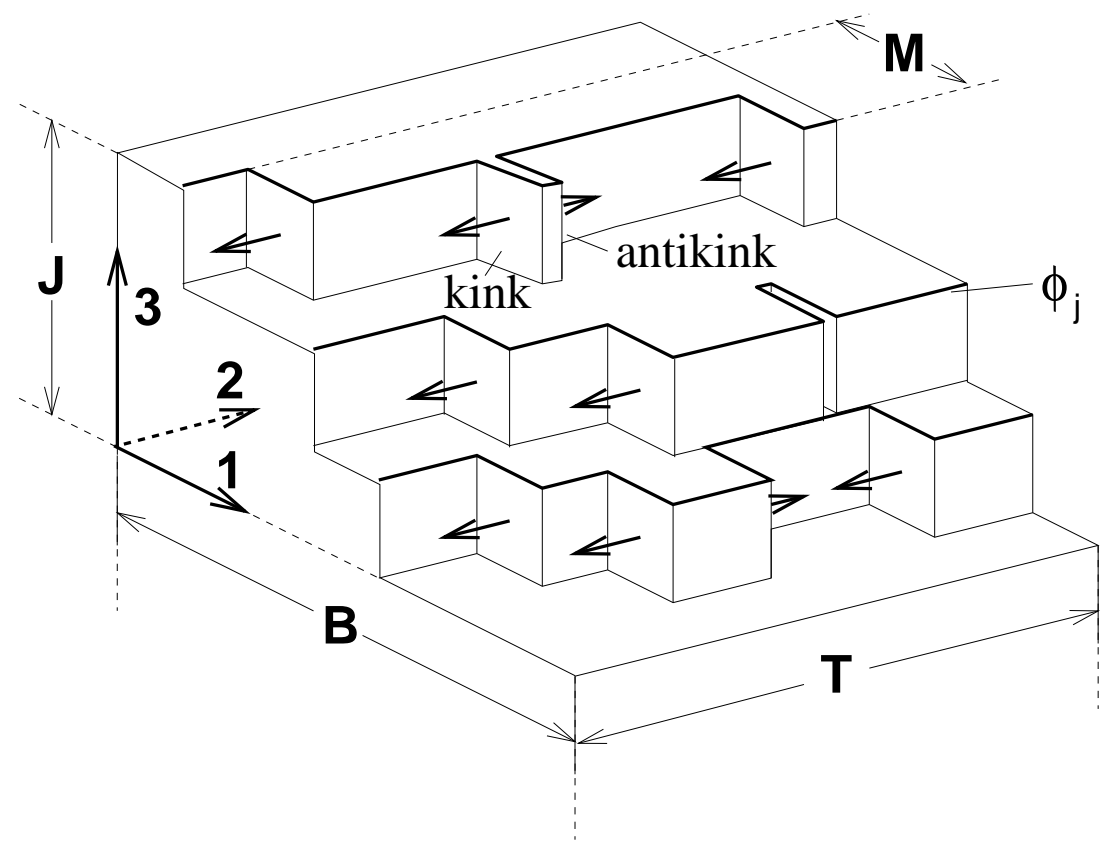

FIG. 1. A piece of the crystalline surface. The deterministic motion is indicated.

Physically we consider a crystal which is in contact with its vapor phase at a pressure slightly higher than equilibrium. Atoms from the gas phase are deposited on the surface. We neglect evaporation. Energetically the fastest process is deposition at (anti)kink sites. In first approximation this leads to a constant speed motion of kinks and antikinks along the steps. The second fastest process is deposition at steps away from kink sites, which is modelled as purely random. Nucleation on the terraces is neglected. For a single height line the dynamics is known as polynuclear growth (PNG) model [8]. To summarize, the surface dynamics has a stochastic and a deterministic piece. In the deterministic part every kink moves with velocity $-w, w>0$, and every antikink with velocity $w$ along the 2-direction. Whenever a kink and antikink pair meet they simply annihilate each other. In addition kink-antikink pairs are randomly generated with uniform rate $\xi$ provided the constraint $\phi_{j}(\tau) \leq \phi_{j+1}(\tau)$ is not violated. This means, if allowed, one has $\phi_{j}\left(\tau_{0}\right) \longrightarrow \phi_{j}\left(\tau_{0}\right)+1$ at some $\tau_{0}$. Under the deterministic motion the newly created kink-antikink pair at $\left(\tau_{0}, \phi_{j}\left(\tau_{0}\right)\right)$ immediately separates with speed $2 w$. Since the higher lying terraces expand at the cost of lower lying ones, the surface grows along the 3 -direction.

In order to determine the steady state we have to work in finite volume with the slope $\mathbf{u}$ imposed by appropriate boundary conditions. This will be done in Section [1]. In Section III we map the height lines to fermionic world lines. Their weight corresponds to noninteracing fermions. However there is a complicating constraint due to the $u_{2}$-tilt of the surface. We compute the free energy and the two point function of the constrained fermionic system. In Section V we relate these results to the growth velocity and the height-height correlations. 


\section{STEADY STATE}

We restrict to periodic surface configurations obeying $\phi_{j+J}(\tau)=\phi_{j}(\tau)+B$ and $\phi_{j}(\tau+T)=\phi_{j}(\tau)+M$, where $T \in \mathbb{R}^{+}, B, J, M \in \mathbb{N}$. This induces the slope $\mathbf{u}=\left(u_{1}, u_{2}\right)$ with $u_{1}=-J / B$ and $u_{2}=-u_{1} M / T$ (see Figure 1). For convenience we choose $u_{1}<0, u_{2}>0$. The remaining inclinations follow by symmetry. In order to determine a surface configuration it then suffices to specify $J$ height lines $\phi_{1}(\tau), \ldots, \phi_{J}(\tau)$ modulo $B$ in the interval $0 \leq \tau \leq T$. The height in the 3-direction is encoded only modulo $J$. Let $x_{l}^{(j)} \in[0, T]$ be the position of the $l$-th kink for height line $j, l=1, \ldots, n^{(j)}+M$ and $y_{l}^{(j)} \in[0, T]$ be the position of the $l$-th antikink, $l=1, \ldots, n^{(j)}$. We set $\mathbf{n}=\left(n^{(1)}, \ldots, n^{(J)}\right)$, $\|\mathbf{n}\|=2\left(n^{(1)}+\cdots+n^{(J)}\right)+M J$, the total number of kinks and antikinks, and $\Phi_{0}=\left(\phi_{1}(0+), \ldots, \phi_{J}(0+)\right)$. The space of height line configurations $\Gamma$ decomposes then as the disjoint union $\Gamma=\dot{U}_{\mathbf{n}, \Phi_{0}} \Gamma\left(\mathbf{n} ; \Phi_{0}\right)$, where $\Gamma\left(\mathbf{n} ; \Phi_{0}\right)$ is some subset of $[0, T]^{\|\mathbf{n}\|}$ as defined through the constraints already explained (we define $[0, T]^{0}$ to be a single point). Furthermore for fixed $\mathbf{n}$ the $\Gamma\left(\mathbf{n} ; \Phi_{0}\right)$ are glued together in a way that is determined by the condition that (anti-)kinks leaving the interval $[0, T]$ reappear smoothly on the opposite side, forming the manifold $\Gamma(\mathbf{n})$ (we avoid the subtleties of defining properly the manifold $\Gamma(\mathbf{n})$ ). The stationary measure $P$ restricted to $\Gamma(\mathbf{n})$ is given by

$$
\left.P\right|_{\Gamma(\mathbf{n})}=p(\|\mathbf{n}\|) \prod_{j=1}^{J}\left(\prod_{l=1}^{n^{(j)}+M} \mathrm{~d} x_{l}^{(j)} \prod_{l=1}^{n^{(j)}} \mathrm{d} y_{l}^{(j)}\right) \quad, \quad p(n)=\eta^{n} / Z .
$$

Here $\eta=\sqrt{\xi / 2 w}$ and the normalization

$$
Z=\sum_{n=0}^{\infty} \eta^{n} \sum_{\mathbf{n}, \Phi_{0} ;\|\mathbf{n}\|=n}\left|\Gamma\left(\mathbf{n} ; \Phi_{0}\right)\right|
$$

with $|\cdot|$ denoting the $\|\mathbf{n}\|$-dimensional volume (for $\|\mathbf{n}\|=0$ we set $\left|\Gamma\left(\mathbf{n} ; \Phi_{0}\right)\right|=1$ ).

To verify the stationarity of $P$ we first note that the Lebesgue measure on $\Gamma(\mathbf{n})$ is left invariant by the deterministic part of the dynamics. At a configuration $\phi$ with a total number of $\|n\|$ kinks and antikinks there is a gain in probability with rate $2 w V_{\phi} p(\|\mathbf{n}\|+2)$ through the annihilation of kink-antikink pairs. Here $V_{\phi}=\sum_{j=1}^{J} \int_{0}^{T} \mathrm{~d} \tau \theta\left(\phi_{j}(\tau)-\phi_{j-1}(\tau)\right)$, $\theta(x)=1$ for $x>0$ and $\theta(x)=0$ otherwise, is the "number" of configurations immediately before the annihilation event which yields $\phi$. The loss in probability occurs with rate $\xi V_{\phi} p(\|\mathbf{n}\|)$ since $V_{\phi}$ is also the "number" of configurations which arise from $\phi$ by generating a kink-antikink pair. Equating gain and loss we obtain (4). For $\eta \neq 0$ the process is ergodic on $\Gamma$ and therefore the stationary measure $P$ is unique. (Any configuration can be evolved to a reference configuration by properly adding kink-antikink pairs in the course of the deterministic evolution).

The measure $P$ and the partition function $Z$ depend on $T, B$ and $J, M$. We want to take the infinite volume limit $B \rightarrow \infty, T \rightarrow \infty$ choosing $J, M$ such that the slope $\mathbf{u}$ is fixed.

\section{FERMI LIQUID WITH CHIRAL BOUNDARY CONDITIONS, FREE ENERGY}

From (4) we see that, subject to the constraint of not crossing, every configuration of height lines has the weight $\eta^{\|\mathbf{n}\|}$. This is precicely the weight of free fermion world lines in the Euclidian (imaginary time) set-up [9]. The surface tilt induces chiral boundary conditions.

Fermion world lines do not overlap. This can be achieved simply by redefining

$$
\omega_{j}(\tau)=\phi_{j}(\tau)+j-1, \quad j=1, \ldots, J
$$

with periodized version

$$
\tilde{\omega}_{j}(\tau)=\omega_{j}(\tau) \bmod \quad N, \quad N=B+J
$$

(see Figure 2). We introduce the relative center of mass

$$
m(\tau)=\int_{0}^{\tau} \mathrm{d} \tau^{\prime} \sum_{j=1}^{J} \dot{\omega}_{j}\left(\tau^{\prime}\right) \quad \in \mathbb{Z}
$$

Clearly $m(\cdot)$ increases by 1 at every kink and decreases by 1 at every antikink. The chiral boundary conditions are then

$$
m(T)=J M \quad, \quad \tilde{\omega}_{j}(T)=\tilde{\omega}_{j}(0)+M \quad \bmod \quad N, \quad j=1, \ldots, J .
$$




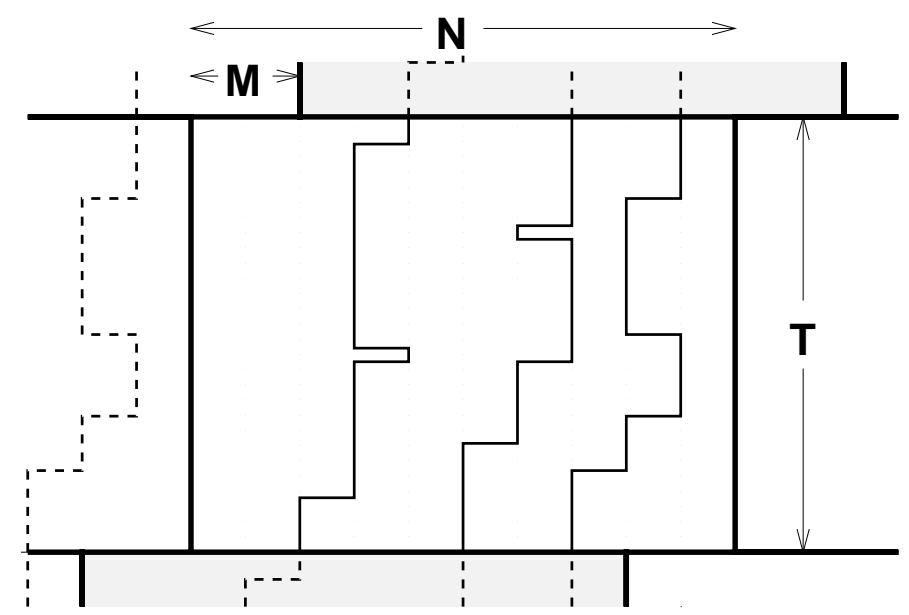

FIG. 2. The mapping of the surface in Figure 1 to fermion world lines. Left and right boundaries are identified. The upper and lower boundaries are identified modulo a relative shift of $M$ sites.

The Fermi liquid lives on $\{1, \ldots, N\}$. Let $\left\{a_{x}^{+}, a_{x}\right\}$ be the corresponding Fermi field operators. They act on the fermion Fock space $\mathcal{F}$, which is spanned by $\left\{a_{x_{1}}^{+} \ldots a_{x_{l}}^{+}|\Omega\rangle\right\}$ with $|\Omega\rangle$ the Fock vacuum. Since $m(\tau) \in \mathbb{Z}$, we introduce the auxiliary Hilbert space $\ell_{2}$ with the standard orthonormal basis $\{|m\rangle, m \in \mathbb{Z}\},|m\rangle=(\ldots, 0,1,0, \ldots)$ at the $m$-th position. On this space let $D$ be the (unitary) right shift, $D|m\rangle=|m+1\rangle$. We define the transfer operator

$$
H=-\eta \sum_{x=1}^{N}\left(a_{x+1}^{+} a_{x} \otimes D+a_{x}^{+} a_{x+1} \otimes D^{-1}\right), \quad a_{N+1}=a_{1},
$$

acting on $\mathcal{F} \otimes \ell_{2}$. It expresses that a (anti)kink is linked to the corresponding change in $m(\tau)$. Let $\left|x_{1}, \ldots, x_{J} ; m\right\rangle=$ $a_{x_{J}}^{+} \ldots a_{x_{1}}^{+}|\Omega\rangle \otimes|m\rangle$ with pairwise distinct $x_{j}$. Then the matrix element

$$
\left\langle y_{1}, \ldots, y_{J} ; m\left|e^{-T H}\right| x_{1}, \ldots, x_{J} ; 0\right\rangle
$$

is given by the sum over all fermion world lines with weight $\eta^{n}, n$ the number of fermion jumps, and subject to no overlap, such that $\tilde{\omega}_{j}(0)=x_{j}, \tilde{\omega}_{j}(T)=y_{j}, j=1, \ldots, J$, and $m(T)=m$. For the partition function $Z$ we need $m(T)=J M$ and $y_{j}=x_{j}+M \bmod N$. A fermion configuration is shifted through the total momentum operator defined by

$$
e^{i P} a_{x} e^{-i P}=a_{x+1} \quad, \quad a_{N+1}=a_{1} .
$$

Then

$$
Z=\left\langle 0\left|\operatorname{tr}_{J}\left[\left(e^{i P} \otimes D^{J}\right)^{-M} e^{-T H}\right]\right| 0\right\rangle
$$

with $\operatorname{tr}_{J}$ the trace over the Fock subspace containing $J$ particles.

By the same argument we obtain for the density correlations

$$
\left\langle\left(a_{x}^{+} a_{x}\right)(\tau) a_{y}^{+} a_{y}\right\rangle=Z^{-1}\left\langle 0\left|\operatorname{tr}_{J}\left[\left(e^{i P} \otimes D^{J}\right)^{-M} e^{-(T-\tau) H} a_{x}^{+} a_{x} e^{-\tau H} a_{y}^{+} a_{y}\right]\right| 0\right\rangle .
$$

In (13) and (14) we take the limit $T \rightarrow \infty$, with $M=\alpha T+o(1) \in \mathbb{Z}$, thereby fixing an average drift $\alpha \in \mathbb{R}$, and subsequently the limit $N \rightarrow \infty$ with $J=\rho N+o(1) \in 2 \mathbb{N}+1$, thus fixing the fermion density $\rho \in[0,1]$. We choose only odd $J$ to simplify notation somewhat later on. We remark that we expect the final result to be independent of the order of limits. However, choices other than the one discussed here look considerably more difficult and we did not persue this issue any further.

Let us proceed with the partition function. $H, P$ and $D$ are diagonalized through Fourier transform. Let $\Lambda=\{1, \ldots, N\}$. Then the dual is $\Lambda^{*}=\left\{-\pi+\frac{2 \pi}{N}, \ldots, \pi-\frac{2 \pi}{N}, \pi\right\}$. For $\mathbb{Z}$ the dual is $[-\pi, \pi]$. We set $a_{k}=N^{-1 / 2} \sum_{x} \exp (-i k x) a_{x}$ for $k \in \Lambda^{*}$ and represent $|m\rangle$ by $\exp (-i m q) \in L^{2}\left([-\pi, \pi],(2 \pi)^{-1} \mathrm{~d} q\right)$. Then

$$
H=-2 \eta \sum_{k \in \Lambda^{*}} \cos (k+q) a_{k}^{+} a_{k}
$$


considered as multiplication operator in $q$ on $L^{2}\left([-\pi, \pi],(2 \pi)^{-1} \mathrm{~d} q\right)$. Also

$$
e^{i P} \otimes D^{J}=\exp \left[-i \sum_{k \in \Lambda^{*}}(k+q) a_{k}^{+} a_{k}\right]
$$

on the $J$-particle subspace. Note that $\left[H, e^{i P} \otimes D^{J}\right]=0$.

We have

$$
Z=\int \frac{d q}{2 \pi} \sum_{k_{1}<\cdots<k_{J}} \exp \left(2 \eta T \sum_{j=1}^{J} \cos \left(k_{j}+q\right)+i M \sum_{j=1}^{J}\left(k_{j}+q\right)\right) .
$$

We write $\sum_{j=1}^{J} \cos \left(k_{j}+q\right)=A_{\{k\}} \cos \left(\phi_{\{k\}}+q\right)$, where $A_{\{k\}}=\left|e^{i k_{1}}+\cdots+e^{i k_{J}}\right|$ and $\phi_{\{k\}}=\arg \left(e^{i k_{1}}+\cdots+e^{i k_{J}}\right)$, and use the integral representation of the modified Bessel function, $I_{n}(x)=\pi^{-1} \int_{0}^{\pi} \mathrm{d} q e^{x \cos (\phi+q)} \cos (n(\phi+q))$ for arbitrary $\phi \in \mathbb{R}$ and integer $n$, to obtain

$$
Z=\sum_{k_{1}<\cdots<k_{J}} \exp \left(-i M\left(k_{1}+\cdots+k_{J}-J \phi_{\{k\}}\right)\right) I_{M J}\left(2 \eta T A_{\{k\}}\right) .
$$

If $A_{\{k\}}$ is maximal, we call $\{k\}$ a ground state mode. By periodicity we have exactly $N$ of them. The contribution of all other modes in (18) is exponentially supressed for large $T$. Thus

$$
Z \simeq N I_{M J}(2 \eta T A)
$$

where $A=\left|\sum_{\kappa=1}^{J} \exp (2 \pi i \kappa / N)\right|=N \sin (\pi \rho) / \pi+o(1)$ for large $N$. The asymptotic expansion for the modified Bessel function,

$$
I_{n}(n / z)=(2 \pi n)^{-1 / 2}\left(z+\sqrt{1+z^{2}}\right)^{-n} \exp \left(n \sqrt{1+1 / z^{2}}\right)\left(1+\mathcal{O}\left(n^{-1}\right)\right),
$$

yields immediately the specific free energy of the fermion system in the infinite volume

$$
f=\lim _{N \rightarrow \infty} \lim _{T \rightarrow \infty}(T N)^{-1} \ln Z=\alpha \rho\left(\eta_{s} / \eta_{a}+\ln \eta-\ln \left(\eta_{s}+\eta_{a}\right)\right)
$$

with $\eta_{a}=\alpha \pi \rho /(2 \sin \pi \rho)=\lim _{T, N \rightarrow \infty} M J / 2 T A$ and $\eta_{s}=\sqrt{\eta^{2}+\eta_{a}^{2}}$. As explained below, $\eta_{s} \pm \eta_{a}$ take the role of effective right and left jump rates for the fermions. As a further consequence of (20) we note the relation

$$
\lim _{n \rightarrow \infty} \frac{I_{n-x}(n / z-c)}{I_{n}(n / z)}=\left(z+\sqrt{1+z^{2}}\right)^{x} \exp \left(-c \sqrt{1+z^{2}}\right)
$$

for later use.

\section{CORRELATIONS}

We turn to the expectation values for density, current, and to their correlations. The operator for the density at $x$ is $\rho_{x}=a_{x}^{+} a_{x}$. To obtain the density at imaginary time $\tau$ we have to evolve $a_{x}^{+} a_{x}$ with the transfer operator $e^{-\tau H}$. The operator for the rightward fermion current from $x$ to $x+1$ is determined through the limit

$$
\begin{aligned}
j_{x}^{+} & =\lim _{t \rightarrow 0} \frac{1}{t}\left(a_{x+1}^{+} a_{x+1} a_{x} a_{x}^{+}\right)(t)\left(a_{x}^{+} a_{x} a_{x+1} a_{x+1}^{+}\right)(0) \\
& =\left.\frac{\partial}{\partial t}\left(a_{x+1}^{+} a_{x+1} a_{x} a_{x}^{+}\right)(t)\left(a_{x}^{+} a_{x} a_{x+1} a_{x+1}^{+}\right)(0)\right|_{t=0} \\
& =\left[a_{x+1}^{+} a_{x+1} a_{x} a_{x}^{+}, H\right] a_{x}^{+} a_{x} a_{x+1} a_{x+1}^{+} \\
& =\eta\left(a_{x+1}^{+} a_{x} \otimes D\right)
\end{aligned}
$$

by the fermion anticommutation rules. Analoguously the operator for the leftward current from $x$ to $x-1, j_{x}^{-}=$ $\eta\left(a_{x-1}^{+} a_{x} \otimes D^{-1}\right)$.

Let us denote $j_{x}^{(+1)}=j_{x}^{+}, j_{x}^{(0)}=\eta \rho_{x}$ and $j_{x}^{(-1)}=j_{x}^{-}$. To determine their expectations, we recall that in the limit $T \rightarrow \infty$ the trace $\operatorname{tr}_{J}$ reduces to the sum $\sum_{\kappa \in \Lambda^{*}}\langle\cdot\rangle_{\kappa}$, where $\langle\cdot\rangle_{\kappa}$ is the expectation value with respect to the $\kappa$-ground 
state $a_{\kappa-\pi(J-1) / N}^{+} \cdots a_{\kappa+\pi(J-1) / N}^{+}|\Omega\rangle$. Because of periodicity these expectation values are invariant under arbitrary translations in time, space, and even in dual space. Thus for $\beta \in\{-1,0,1\}$ we have

$$
\begin{aligned}
\eta^{-1} & \left\langle j_{x}^{(\beta)}(\tau)\right\rangle=\left\langle a_{\beta}^{+} a_{0} \otimes D^{\beta}\right\rangle=N^{-1} \sum_{k k^{\prime}} e^{-i k \beta}\left\langle a_{k}^{+} a_{k^{\prime}} \otimes D^{\beta}\right\rangle= \\
& =\lim _{T \rightarrow \infty} Z^{-1} N^{-1} \sum_{k} \int \frac{\mathrm{d} q}{2 \pi} \sum_{\kappa} e^{2 \eta T A \cos (\kappa+q)+i M J(\kappa+q)} e^{-i(k+q) \beta}\left\langle a_{k}^{+} a_{k}\right\rangle_{\kappa} \\
& =\lim _{T \rightarrow \infty} N^{-1} \sum_{k} \sum_{\kappa} e^{-i(k-\kappa) \beta}\left\langle a_{k}^{+} a_{k}\right\rangle_{\kappa} Z^{-1} \int \frac{\mathrm{d} q}{2 \pi} e^{2 \eta T A \cos (\kappa+q)+i(M J-\beta)(\kappa+q)} \\
& =\lim _{T \rightarrow \infty} N^{-1} \sum_{k} e^{-i k \beta}\left\langle a_{k}^{+} a_{k}\right\rangle_{0} I_{M J-\beta}(2 \eta T A) / I_{M J}(2 \eta T A) \\
& =\left(\alpha J / 2 \eta A+\sqrt{1+(\alpha J / 2 \eta A)^{2}}\right)^{\beta} N^{-1} \sum_{k} e^{-i k \beta}\left\langle a_{k}^{+} a_{k}\right\rangle_{0} .
\end{aligned}
$$

In the limit $N \rightarrow \infty$ we obtain

$$
\left\langle\rho_{x}(\tau)\right\rangle=\rho
$$

and

$$
\left\langle j_{x}^{ \pm}(\tau)\right\rangle=\frac{1}{2}\left(\sqrt{(\alpha \rho)^{2}+(2 \eta \sin \pi \rho / \pi)^{2}} \pm \alpha \rho\right) .
$$

We define the structure function

$$
S^{\beta \beta^{\prime}}(x, \tau)=\eta^{-2}\left\langle j_{x}^{(\beta)}(\tau) j_{0}^{\left(\beta^{\prime}\right)}(0)\right\rangle-\left\langle j_{x}^{(\beta)}(\tau)\right\rangle\left\langle j_{0}^{\left(\beta^{\prime}\right)}(0)\right\rangle
$$

in the infinite volume limit. Assuming $\tau>0$ and using the same method as above, we obtain for finite $N$

$$
\begin{aligned}
S_{N}^{\beta \beta^{\prime}}(x, \tau)= & N^{-2} \sum_{k \kappa k^{\prime} \kappa^{\prime}} e^{-i(k-\kappa) x-i\left(k \beta+k^{\prime} \beta^{\prime}\right)}\left\langle\left(a_{k}^{+} a_{\kappa} \otimes D^{\beta}\right)(\tau)\left(a_{k^{\prime}}^{+} a_{\kappa^{\prime}} \otimes D^{\beta^{\prime}}\right)(0)\right\rangle \\
& -\left\langle j_{x}^{(\beta)}(\tau)\right\rangle\left\langle j_{0}^{\left(\beta^{\prime}\right)}(0)\right\rangle \\
= & N^{-2} \sum_{k k^{\prime}} e^{-i\left(k-k^{\prime}\right) x-i\left(k \beta+k^{\prime} \beta^{\prime}\right)}\left\langle\left(a_{k}^{+} a_{k^{\prime}} \otimes D^{\beta}\right)(\tau)\left(a_{k^{\prime}}^{+} a_{k} \otimes D^{\beta^{\prime}}\right)(0)\right\rangle \\
= & \lim _{T \rightarrow \infty} Z^{-1} N^{-2} \sum_{k k^{\prime}} \sum_{\kappa} \int \frac{\mathrm{d} l}{2 \pi} e^{-i\left(k-k^{\prime}\right) x-i\left((k+q) \beta+\left(k^{\prime}+q\right) \beta^{\prime}\right)} \times \\
& \times e^{2 \eta T A \cos (\kappa+q)-2 \eta \tau\left(\cos (k+q)-\cos \left(k^{\prime}+q\right)\right)+i M J(\kappa+q)}\left\langle a_{k}^{+} a_{k^{\prime}} a_{k^{\prime}}^{+} a_{k}\right\rangle_{\kappa} \\
= & \lim _{T \rightarrow \infty} Z^{-1} N^{-2} \sum_{k k^{\prime}} \sum_{\kappa} e^{-i\left(k-k^{\prime}\right) x-i\left((k-\kappa) \beta+\left(k^{\prime}-\kappa\right) \beta^{\prime}\right)}\left\langle a_{k}^{+} a_{k}\right\rangle_{\kappa}\left\langle a_{k^{\prime}} a_{k^{\prime}}^{+}\right\rangle_{\kappa} \times \\
& \times \int_{\frac{\mathrm{d}}{2 \pi} l} e^{2 \eta T(A-\Delta A) \cos (\kappa-\Delta \kappa+q)+i\left(M J-\beta-\beta^{\prime}\right)(\kappa+q)} \\
= & \lim _{T \rightarrow \infty} N^{-3} \sum_{\kappa} \sum_{k k^{\prime}} e^{-i\left(k-k^{\prime}\right) x-i(k-\kappa) \beta-i\left(k^{\prime}-\kappa\right) \beta^{\prime}}\left\langle a_{k}^{+} a_{k}\right\rangle_{\kappa}\left\langle a_{k^{\prime}} a_{k^{\prime}}^{+}\right\rangle_{\kappa} \times \\
& \times e^{-i\left(M J-\beta-\beta^{\prime}\right)(\Delta \kappa)} I_{M J-\beta-\beta^{\prime}}(2 \eta T(A-\Delta A)) / I_{M J}(2 \eta T A) \\
= & N^{-2} \sum_{k k^{\prime}} e^{-i\left(k-k^{\prime}\right) x-i\left(k \beta+k^{\prime} \beta^{\prime}\right)}\left\langle a_{k}^{+} a_{k}\right\rangle_{0}\left\langle a_{k^{\prime}} a_{k^{\prime}}^{+}\right\rangle_{0}\left(\alpha J / 2 \eta A+\sqrt{1+(\alpha J / 2 \eta A)^{2}}\right)^{\beta+\beta^{\prime}} \times \\
& \times \exp \left[-2 \eta \tau \sqrt{1+(\alpha J / 2 \eta A)^{2}}\left(\cos k-\cos k^{\prime}\right)-i \tau \alpha J\left(\sin k-\sin k^{\prime}\right) / A\right]
\end{aligned}
$$

with $\Delta A=(\tau / T)\left(\cos (k-\kappa)-\cos \left(k^{\prime}-\kappa\right)\right)+\mathcal{O}\left(N^{-1} T^{-2}\right), \Delta \kappa=(\tau / T A)\left(\sin (k-\kappa)-\sin \left(k^{\prime}-\kappa\right)\right)+\mathcal{O}\left(N^{-2} T^{-2}\right)$. In the last step we used once more (22) and shifted $k$ and $k^{\prime}$ by $\kappa$. Taking the limit $N \rightarrow \infty$ results in

$$
\begin{aligned}
& S^{\beta \beta^{\prime}}(x, \tau)=\left(\left(\eta_{a}+\eta_{s}\right) / \eta\right)^{\beta+\beta^{\prime}} \int_{-\pi \rho}^{\pi \rho} \frac{\mathrm{d} k}{2 \pi} \int_{\pi \rho}^{2 \pi-\pi \rho} \frac{\mathrm{d} k^{\prime}}{2 \pi} \\
& \quad \exp \left[-2 \tau\left(\eta_{s}\left(\cos k-\cos k^{\prime}\right)+i \eta_{a}\left(\sin k-\sin k^{\prime}\right)\right)-i\left(k-k^{\prime}\right) x-i\left(k \beta+k^{\prime} \beta^{\prime}\right)\right]
\end{aligned}
$$


for $\tau>0$ and $S^{\beta \beta^{\prime}}(x, \tau)=S^{\beta \beta^{\prime}}(-x,-\tau)$ by symmetry. The Fourier transform of $S^{\beta \beta^{\prime}}$ with respect to $x$ equals

$$
\begin{aligned}
& \hat{S}^{\beta \beta^{\prime}}(K, \tau)=\left(\left(\eta_{a}+\eta_{s}\right) / \eta\right)^{\beta+\beta^{\prime}} \times \\
& \times \int_{|(K / 2)-\pi \rho|}^{(K / 2)+\pi \rho} \frac{\mathrm{d} k}{2 \pi} \exp \left[-4 \tau \sin (K / 2)\left(\eta_{s} \sin k-i \eta_{a} \cos k\right)-i k\left(\beta+\beta^{\prime}\right)-i K\left(\beta-\beta^{\prime}\right) / 2\right]
\end{aligned}
$$

for $K \geq 0$ and $\hat{S}^{\beta \beta^{\prime}}(K, \tau)=\hat{S}^{\beta \beta^{\prime}}(-K, \tau)^{*}$ for $K<0$. For small $|K|$ we have $\hat{S}_{K}^{\beta \beta^{\prime}}(\tau) \propto|K|+\mathcal{O}\left(K^{2}\right)$, and therefore the correlations decay as $\hat{S}_{x}^{\beta \beta^{\prime}}(\tau) \sim x^{-2}$ for large $x$ and constant $\tau$. We remark that the same expectation values and two-point correlations are obtained for a pure Fermi liquid with the usual periodic boundary conditions but with a non-hermitian transfer operator $H=\sum_{x}\left(\eta_{s}+\eta_{a}\right) a_{x+1}^{+} a_{x}+\left(\eta_{s}-\eta_{a}\right) a_{x}^{+} a_{x+1}$.

\section{SURFACE FLUCTUATIONS}

The growth velocity $v$ of the surface in the 3 -direction is proportional to $n$, the total number of kinks and antikinks in $\{1, \ldots, B\} \times[0, T]$,

$$
v=w(B T)^{-1}\langle n\rangle .
$$

In the fermion picture $n$ equals the integrated, total leftward and rightward fermion current, i.e. $n=$ $\sum_{x=1}^{N} \int_{0}^{T} \mathrm{~d} \tau\left(j_{x}^{+}(\tau)+j_{x}^{-}(\tau)\right)$. Thus, using (26),

$$
\begin{aligned}
v & =w(B+J) B^{-1} \sqrt{(2 \eta \sin \pi \rho / \pi)^{2}+(\alpha \rho)^{2}} \\
& =w \frac{1+\left|u_{1}\right|}{\pi} \sqrt{\left(2 \eta \sin \frac{\pi\left|u_{1}\right|}{1+\left|u_{1}\right|}\right)^{2}+\left(\frac{\pi\left|u_{2}\right|}{1+\left|u_{1}\right|}\right)^{2}},
\end{aligned}
$$

because the surface inclination $\mathbf{u}$ is related to the fermion density $\rho$ and drift $\alpha$ as $\rho=\left|u_{1}\right| /\left(1+\left|u_{1}\right|\right)$ and $\alpha=\left|u_{2} / u_{1}\right|$. By symmetry (32) holds for arbitrarty $\mathbf{u} \in \mathbb{R}^{2}$. As can be seen from Figure 3, for small $\mathbf{u}$ we have qualitatively $v(u) \simeq|\mathbf{u}|$, which is characteristic for models ignoring nucleation and island formation on terraces. Except for $\mathbf{u}=(0,0)$ the growth velocity is saddlelike, i.e. $\operatorname{det} \partial^{2} v \leq 0$. Thus the RG analysis of Wolf applies with the prediction that the Gates-Westcott crystal surface is in the Edwards-Wilkinson universality class. If so, the surface fluctuations should increase logarithmically for large separation.

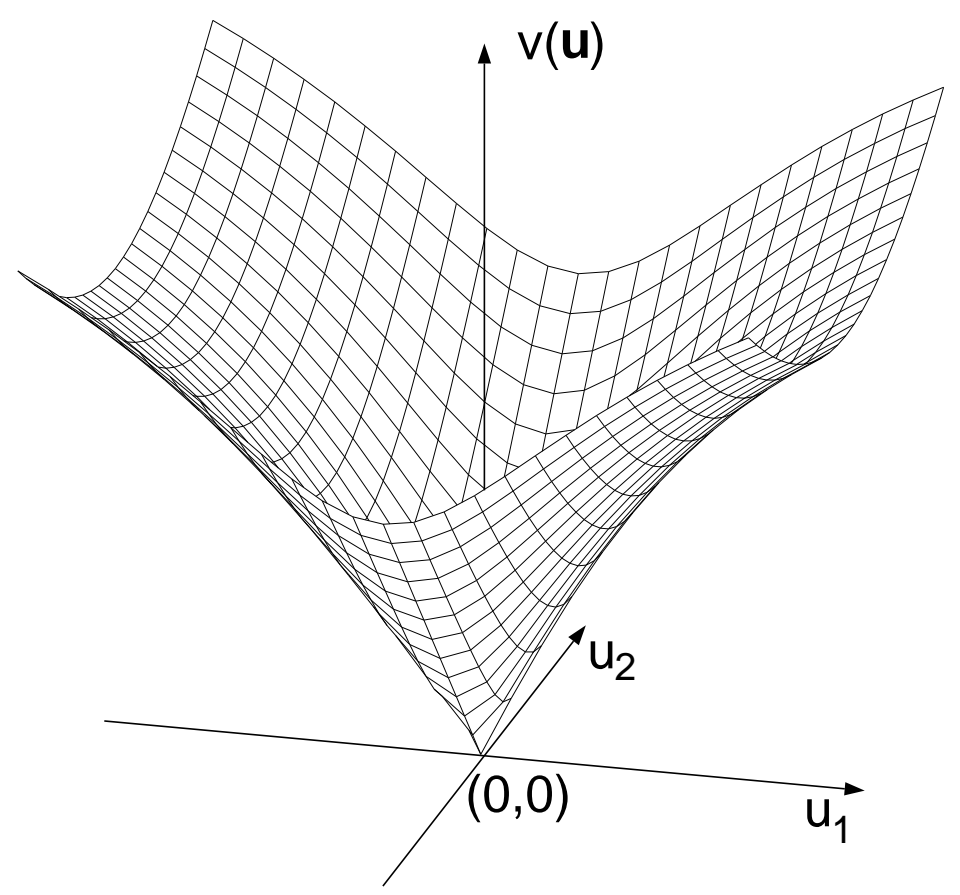

FIG. 3. The growth velocity $v$ as a function of the surface tilt $\mathbf{u}$. 
To check this property for the Gates-Westcott model we parametrize the surface relative to the new plane obtained by rotating the 1-2-plane by $45^{\circ}$ around the 2 -axis. The new height function $\tilde{h}$ is then given implicitly for each $\tau$ by $\tilde{h}(x, \tau)=h(m, \tau)-m$ where $x=h(m, \tau)+m . \tilde{h}$ is more explicitly related to the fermion world lines $\omega_{x}(\tau)$, since

$$
\tilde{h}(x, \tau)=\tilde{h}(0, \tau)+\sum_{y=1}^{x} \omega_{y}(\tau) .
$$

The height difference between $(0,0)$ and $(x, t), \Delta(x, t)=\tilde{h}(x, t)-\tilde{h}(0,0)$, equals the number of fermion lines passing between $(0,0)$ and $(x, t)$. We denote the number of fermions at time $t$ in $\{1, \ldots, x\}$ as $N_{[1, x]}(t), N_{[y, x]}(t)=\sum_{x^{\prime}=y}^{x} \rho_{x^{\prime}}(t)$, and the integrated fermion current from site $x$ to site $x+1$ during the time interval $[0, t]$ as $J_{[0, t]}(x), J_{[0, t]}(x)=$ $\int_{0}^{t} \mathrm{~d} \tau\left(j_{x}^{+}(\tau)-j_{x}^{-}(\tau)\right)$. The height difference $\Delta(x, t)$ is then expressed as

$$
\Delta(x, t)=N_{[1, x]}(0)-J_{[0, t]}(x) .
$$

The mean height difference is given by

$$
\langle\Delta(x, t)\rangle=\rho(x-\alpha t) .
$$

To compute the height-height correlations we use fermion particle conservation

$$
J_{[0, t]}(x)-J_{[0, t]}(y)=N_{[y+1, x]}(0)-N_{[y+1, x]}(t)
$$

for $x>y$. Then, using that current and density correlations decouple for large distances, we have

$$
\begin{aligned}
\left\langle\Delta(x, t)^{2}\right\rangle- & \langle\Delta(x, t)\rangle^{2}= \\
= & \left\langle\left(N_{[1, x]}(0)-J_{[0, t]}(x)\right)^{2}\right\rangle-(\rho x-\alpha \rho t)^{2} \\
= & \lim _{y \rightarrow \infty}\left\langle\left(N_{[1, x]}(0)-J_{[0, t]}(x)+J_{[0, t]}(-y)\right) \times\right. \\
& \left.\times\left(N_{[1, x]}(0)-J_{[0, t]}(x)+J_{[0, t]}(y)\right)\right\rangle-\rho^{2} x^{2} \\
= & \lim _{y \rightarrow \infty}\left\langle\left(N_{[-y+1, x]}(t)-N_{[-y+1,0]}(0)\right)\left(N_{[1, y]}(0)-N_{[x+1, y]}(t)\right)\right\rangle-\rho^{2} x^{2} \\
= & \lim _{y \rightarrow \infty} \int \frac{\mathrm{d} k}{2 \pi} \frac{e^{i k(y-1)}-e^{i k(x+1)}}{1-e^{-i k}}\left(\frac{e^{i k}-e^{i k(y+1)}}{1-e^{i k}} \hat{S}(k, t)-\right. \\
& \left.-\frac{e^{-i k(x+1)}-e^{i k(y+1)}}{1-e^{i k}} \hat{S}(k, 0)\right)-\frac{e^{i k(y-1)}-e^{-i k}}{1-e^{-i k}} \times \\
& \times\left(\frac{e^{i k}-e^{i k(y+1)}}{1-e^{i k}} \hat{S}(k, 0)-\frac{e^{-i k(x+1)}-e^{i k(y+1)}}{1-e^{i k}} \hat{S}(k,-t)\right) \\
= & \int \frac{\mathrm{d} k}{2 \pi} \frac{1}{1-\cos k}\left(\hat{S}(k, 0)-e^{-i k x} \hat{S}(k, t)\right),
\end{aligned}
$$

with $\hat{S}=\hat{S}^{00}$. The rapidly oscillating terms vanish in the limit $y \rightarrow \infty$, since they are multiplied by continuous $2 \pi$-periodic functions. For large $|t|$, keeping $x / t$ constant, the leading contribution is

$$
\begin{aligned}
\left\langle\Delta(x, t)^{2}\right\rangle & -\langle\Delta(x, t)\rangle^{2}= \\
& =\pi^{-2} \int_{0}^{\pi|t|} \frac{\mathrm{d} k}{k}\left(1-e^{-2 k \eta_{s} \sin \pi \rho} \cos k\left(2 \eta_{a} \cos \pi \rho-x / t\right)\right)+\mathcal{O}(1) \\
& =\pi^{-2} \ln (|t|)+\mathcal{O}(1) .
\end{aligned}
$$

If $|x|$ gets large while $t=o(x)$ we have

$$
\begin{aligned}
\left\langle\Delta(x, t)^{2}\right\rangle & -\langle\Delta(x, t)\rangle^{2}= \\
& =\pi^{-2} \int_{0}^{\pi|x|} \frac{\mathrm{d} k}{k}\left(1-e^{-2 k|t / x| \eta_{s} \sin \pi \rho} \cos k\left(2(t / x) \eta_{a} \cos \pi \rho-1\right)\right)+\mathcal{O}(1) \\
& =\pi^{-2} \ln (|x|)+\mathcal{O}(1) .
\end{aligned}
$$

In conclusion we have shown that indeed the height-height correlations increase logarithmically with separation. Properly speaking the fluctuations should be considered in the same reference frame as the growth velocity. However, this will not change their logarithmic growth. 


\section{ACKNOWLEDGEMENTS}

We thank J. Krug for insisting on arbitrary tilts.

[1] M. Kardar, G. Parisi, and Y.-C. Zhang, Phys. Rev. Lett. 56, 889 (1986)

[2] J. Krug and H. Spohn, in Solids Far From Equilibrium, C. Godrèche, ed. (Cambridge Univ. Press, Cambridge, 1991)

[3] A.-L. Barabasi and H. E. Stanley, Fractal Concepts in Surface Growth (Cambridge Univ. Press, Cambridge, 1995)

[4] T. Halpin-Healy and Y.-C. Zhang, Phys. Rep. 254, 215 (1995)

[5] D. E. Wolf, Phys. Rev. Lett. 67, 1783 (1991)

[6] D. J. Gates and M. Westcott, J. Stat. Phys. 81, 681 (1995)

[7] H. Spohn, in Hydrodynamic Behavior and Interacting Particle Systems, G. Papanicolaou, ed. (Springer Verlag, 1987)

[8] J. Krug and H. Spohn, Europhys. Lett. 8, 219 (1989)

[9] P. Bak and J. Villain, J. Physique 42, 657 (1981)

[10] M. Abramowitz and I. A. Stegun Pocketbook of Mathematical Functions (Verlag Harri Deutsch, Frankfurt am Main, 1984) 